\title{
La propagation des ondes de marée ${ }^{(*)}$
}

\section{The propagation of tide waves $^{(*)}$}

\author{
PAR L. VANTROYS \\ CHEF DU SERVICE DES USINES MAREMIOTRICES \\ A L'BLECTHICTÉ DE FRANCE
}

Les hypotheses simplificatrices ayant permis d'aborder le probleme de la propagation des ondes de marée sont mises en évidence:

- Ce sont des «ondes longues» ou «ondes de masse $\$$, c'est-didire que (contrairement at cas de l'étude de la houle) on y néglige les gradients verticaux;

- Les oscillations de la surface libre sont in/iniment petites par rapport a la profondear mogenne:

- Les oscillations étudiées sont rigoureusement sinusoz̃dales ce qui en permet la représentation par des nombres complexes.

Les hypothèses complémentaires définissent les differents cas étudies :

1. Zone géographique restreinte, profondeur uniforme, rotation uniforme, absence de frottement, absence de potentiel générateur.

2. Zone géographique restreinte, profondeur uniforme, rotation uniforme, frottement fonction linéaire de la vitesse du courant, absence de potentiel génératenr.

3. (Cas des marées littorales). Zone géographique restreinte, profondeur non uniforme, rotation uniforme, frottement, absence de potentiel générateur.

4. (Cas des marées océaniques). Zone géographique étendue, profondeur non uniforme, rotation non uniforme, absence de frottement, action da potentiel génératenr.

Des développements mathématiques représentant tes solutions des cas 1 et 2 ont pu etre explicités. Par contre, pour les cas plus complexes 3 et 4 (profondeur non uniforme), $\dot{a}$ défaut de formule générale, des méthodes d'approximation ont été indiquées permettant une solution des problèmes rencontrés par des méthodes numériques ou par l'emploi de modèles réduits hydrauliques.

(*) Cette communication présente sous une forme condensée quelques résultats exposés dans une thèse de Doctorat es Sciences soutenue en Sorbonne le 23 février 1957 sous le titre «Le remous d'un ourrage dans une mex à marée $\$$.
The following assumptions used in dealing wilh the problem of lide wave propagation are brought into evidence :

a) These waves are «long waves», so that their vertical gradients are neglected, (which is contrury to studies concerning swell);

b) The free surface oscillations are infinitely small, compared to the mean depth;

c) The oscillations observed are strictly sinusoidal, so that they can be represented by complex numbers.

The complementary hypotheses define the various cases considered, as follows :

1. Restricted geographical area, nniform depth, uniform rofation, no friction, no generating potential;

2. Restricted geographical area, uniform depth, uniform rotation, friction depending linearly on stream velocity, no generating potential;

3.(Constal tide case). Restricted geographical area, non-aniform depth, uniform rotation, friction, no generating potential;

8. (Ocean tide case). Large geographical area, non-uniform depth, non-uniform rotation, no friction, actioe generating potential.

Mathematical developments representing solutions for cases 1 and 2 are explicited, but, in the absence of a general formula, approximation methods are given for the more complex. cases 3 and 4 (non-uniform depth), by meuns of which encountered problems can be solved by numerical methods or hydraulic scale models.

( $\left.{ }^{\star}\right)$ This article gives a condensed account of a few results presented in a Doctorat es Sciences thesis read before the Sorbonne on the 23rd february 1957 and entitled «The disturbances caused by a structure in tidal sea $\gg$. 


\section{I. - EQUATIONS DU PROBLÈME}

Reprenons la genèse des équations qui sont à la base de la théorie des marées. Nous pourrons mieux ainsi peser les hypothèses dont dépend leur validité.

Compris entre l'atmosphère, dont la sépare sa surface libre, et l'écorce terrestre, sur laquelle elle repose, l'hydrosphère est susceptible de mouvements divers que la Mécanique classique détermine par l'ensemble des relations suivantes :

Entre fond et surface libre, la masse d'eau des océans et des mers, supposée incompressible, obéit à l'équation dynamique et à la condition de continuité qui, rapportées à des repéres liés à la Terre, s'écrivent :

$$
\begin{gathered}
\frac{\overrightarrow{d V}}{d t}+2 \vec{\omega} \Lambda \vec{V}+\vec{f}(\vec{V})=\underset{\operatorname{grad}}{\vec{g}}\left(-\frac{p}{p}+\mathrm{T}+\mathrm{P}\right) \\
\operatorname{div} \overrightarrow{\mathrm{V})}=0
\end{gathered}
$$

$t=$ variable $《$ temps $»$,

$\vec{V}=$ vitesse du couranl,

$\vec{\omega} \quad=$ vecteur rotation de la Terre,

$\vec{f}(\vec{V})=$ fonctionnelle du vecteur $\vec{V}$ figurant l'action globale des frottements, de la viscosité et de la turbulence,

$p=$ pression,

u. = masse spécifique de l'eau de mer,

$\mathrm{T}=$ potentiel du champ permanent de la pesanteur $\left(^{*}\right)$,

$\mathrm{P}=$ potentiel du champ perturbateur, générateur de la marée $\left(^{\star *}\right)$.

Sur le fond, le vecteur courant devra rester tangent à la surface limite, que nous suppose-

(*) Ce potentiel $T$ comprend l'attraction résultante de toutes les masses terrestres supposées rigidement liées les unes aux autres - l'hydrosphère étant en équilibre - et l'action de la force centrifuge due à la rotation de la Terre. T est indépendant de la variable $t$.

$\left({ }^{*}\right)$ Ce potentiel $\mathrm{P}$ comprend, rapportées à des repères liés à la Terre, les attractions lunaire et solaire et l'attraction 《 du bourrelet », qui est la différence, due à la marée elle-même, entre l'attraction instantanée des forces terrestres et l'attraction permanentc qui entre dans la définition de $T$; cette différence est égale à l'attraction du bourrelet dit « positif » diminuée de l'attraction du bourrelet dit « négatif 》; le bourrelet 《 positif » est constitué par la masse d'eau qui s'est élevée au-dessus de la surface libre d'équilibre dans le champ dérivant de $T$, le bourrelet «négatif » étant la masse d'eau qui serait nécessaire pour combler toutes les dépressions où la surface libre s'est abaissée au-dessous de cette situation d'équilibre. $\mathrm{P}$ est fonction du temps $t$. rons fixe, ce qui revient à négliger toute déformation de l'écorce terrestre, et en particulier les marćes de l'écorce terrestre. Cette condition s'écrira :

$$
\vec{n} \cdot \vec{V}=0
$$

$\vec{n}$ étant le vecteur unitaire normal au fond.

Sur la surface libre, il devra y avoir équilibre de pression entre hydrosphère et atmosphìre. Si nous négligeons la réaclion sur l'océan des perturbations méterologiques, el en particulier de la marée atmosphérique, nous pourrons écrire que la pression à la surface libre est uniforme et constante :

$$
p=p_{i}
$$

L'ensemble des équations (D), (C), (F), (S), et de conditions initiales à préciser, definit le mouvement de l'hydrosphère.

Si l'on n'a en vue que l'étude des marées, diverses hypothèses permettent de simplifier les relations ci-dessus.

Tout d'abord, supposant faibles les amplitudes des déplacements et des courants, on négligera, devant ceux du premier degre, les ter. mes du second degré par rapport au courant el à ses dérivées; d'où :

$$
\frac{d \vec{V}}{d t}=\frac{\partial \vec{V}}{\partial t}+\vec{V} \cdot \overrightarrow{\operatorname{grad}}(\vec{V}) \# \frac{\partial \vec{V}}{\partial t}=\lambda \vec{V}
$$

le symbole $\lambda$ représentant l'opération de dérivation par rapport au temps, les variables spatiales restant constantes.

Une remarque essentielle s'impose, à savoir que le champ perturbateur (variable) qui dérivo de $\mathrm{P}$ est extrêmement petit à côté du champ? permanent qui dérive de $\mathrm{T}$; le rapport du premier au second est de lordre de $10^{-7}$. Il en résulte que l'efrel de ce champ perturbateur restera faible dans la direction du champ permam nent (c'est-à-dire verticalement), mais pourra, par contre, acquérir de grandes amplitudes parallèlement aux surfaces de niveau du champ permanent (c'est-à-dire horizontalement). C'est ce qu'on constate en fait dans lobservation des marées. Ainsi, en Manche, pour des dénivellations de l'ordre de 10 mètres, on a des déplacements horizontaux de masses d'eau qui dépassent 10 kilomètres.

Dans le domaine du calcul, ceci se traduit par la présence dans la même équation (D) de termes très grands à côté de termes très petits. Fort heureusement, la décomposition de cette 
equation vectorielle en trois équations scalaires va nous permettre de ne faire figurer le champ permanent que dans une seule équation, et de l'éliminer complètement des denx antres. Il nous suffira pour cela de projeter l'équation (D) d'une part sur la verticale ascendante du lieu, soit Mz, d'autre part sur deux directions horizontales rectangulaires, soient $\mathrm{MX}^{\prime}$, $\mathrm{MY}^{\prime}$, respectivement tangentes aux courbes $x=\mathrm{C}^{\text {le }}$ et $y=\mathrm{C}^{\text {te }} \mathrm{qui}$ passent par le point $M, x$ et $y$ ctant les coordonnées rectangulaires dans le plan xoy de limage

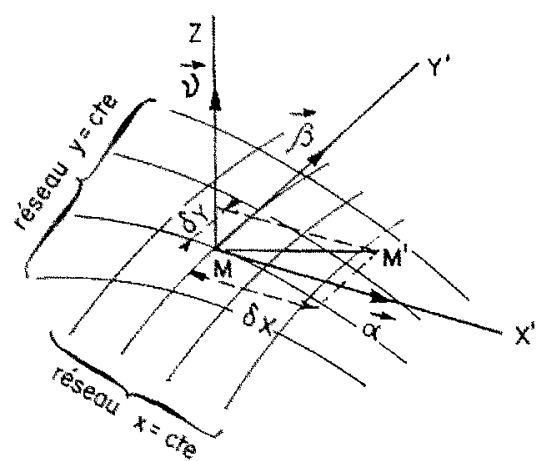

ticale $w$ de la vitesse $\vec{V}$ devant ses composantes horizontales $u, v$, et en assimilant l'effet des frottements à une résistance proportionnelle à la vilesse, c'est-à-dire en prenant $f_{1}=o u, f_{2}=p v$. Pour simplifier l'écriture, nous poserons $2 \overrightarrow{(\omega} \cdot \overrightarrow{\nu)}=\gamma$, coefficient de Coriolis, double de la composante verticale de la rotation terrestre. Nous obtenons ainsi :

$$
\begin{array}{ll}
1 / k[(\lambda+\rho) u-\gamma v]+\left(p_{x} / \mu\right)=\mathrm{P}_{x} & \left(\mathrm{D}_{1}\right)^{\prime} \\
1 / k[\gamma u+(\lambda+\rho) v]+\left(p_{y} / \mu\right)=\mathrm{P}_{y} & \left(\mathrm{D}_{2}\right)^{\prime}
\end{array}
$$

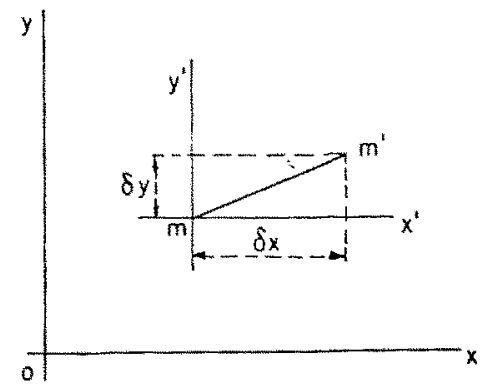

FIs. 1.

cartographique $m$ du point $M$ du géöde dans un système de représentation conforme (d'ailleurs queleonque) dont l'échelle locale est $\left(\mathrm{mm}^{\prime} / \mathrm{MM}^{\prime}\right)=(\delta x / \delta \mathrm{X})=(\delta y / \delta \mathrm{Y})=k(x, y)$, relation qui entraine la correspondance fonctionnelle $(\partial / \partial \mathrm{X}) \equiv k(\partial / x),(\partial / \partial Y)=k(\partial / \partial y)$.

Soient $\vec{\alpha}, \vec{\beta}, \vec{v}$ les veeteurs unitaires portés par les axes MX', MY', MZ. L'équation (D) se décompose ainsi en :

$$
\begin{aligned}
& \lambda u-2 \overrightarrow{(\omega \cdot v)} v+2 \overrightarrow{(\omega} \cdot \vec{\beta}) \cdot w+f_{1}(n, v, w) \\
& =k\left(-p_{k} / \mu\right)+P_{n} \\
& \lambda v-2(\vec{w} \cdot \vec{x}), w+2 \vec{w} \vec{w} \cdot \vec{v}, n+f,(u, v, w) \\
& =k\left[\left(-p_{y} / \mu\right)+P_{y}\right]
\end{aligned}
$$

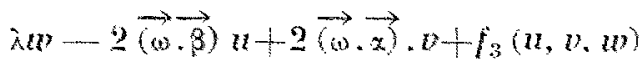

$$
\begin{aligned}
& =\left(-p_{z} / \mu\right)+\mathbf{T}_{Z}+\mathbf{P}_{z}
\end{aligned}
$$

avec:

$$
\vec{V}=\vec{x}, u+\vec{\beta} \cdot \vec{x}+\vec{x} \cdot u
$$

$L_{4}$ importance de $\mathrm{T}_{z}$ à eoté de $\mathrm{P}_{\mathrm{z}}$ et des termes en $u, v, w$, permet - tout en conservant une très grande préeision - de réduire l'équation $\left(\mathrm{D}_{3}\right)$ à ses termes prineipaux, soit :

$$
\left(p_{z} / g\right)=\mathrm{T}_{z}(=-g)
$$

Quant aux equations $\left(D_{2}\right)$ et $\left(D_{2}\right)$, nous les schématiserons en nigligeant lat composante ver-
Revenons à l'équation $\left(\mathrm{D}_{3}\right)^{\prime}$, qui exprime le fait que la répartition des pressions est hydrostatique. Intégrée, cette équation peut s'écrire :

$$
p=p(x, y, t)-g_{u} . Z
$$

Soient $Z=\zeta(x, y, t)$ et $Z=-h(x, y)$ les équations de la surface libre (fluctuante) et du fond (fixe). La relation (D) ${ }_{3}$ "étant vérifiće, à la limite, sur la surface libre, nous aurons :

doù :

$$
p_{0}=p(x, y, t)-g_{u . \zeta}(x, y, t)
$$

$$
p_{x}=g_{\mu} . \zeta_{x}, \quad p_{y}=g_{\mu} \cdot \zeta_{y}
$$

Posons $\mathrm{P}=g_{\varepsilon}$ et portons dans $\left(\mathrm{D}_{1}\right)^{\prime}$ et $\left(\mathrm{D}_{2}\right)^{\prime}$ qui deviennent :

$$
\begin{aligned}
& k g\left(\zeta_{x}-\varepsilon_{x}\right)+(\lambda+o) u-\vartheta v=0 \\
& k g\left(\zeta_{y}-\varepsilon_{y}\right)+\varphi u+(\lambda+\vartheta) v=0
\end{aligned}
$$

Par ailleurs, la condition (F) s'écrit :

$$
k\left(h_{, r} u+h_{y,} p\right)+w(x, y,-h)=0
$$

et d'autre part, près de la surface libre, nous avons :

$$
w(x, y, 0)=\lambda \zeta
$$

Le gradient vertical de la vitesse verticale, entre la surface et le fond, a ainsi pour valeur movenne:

$$
\frac{w(x, y, 0)-w(x, y \cdot-h)}{h}=w_{Z}=\frac{1}{h}\left(\lambda \zeta+k\left(h_{x} u+h_{y} v\right)\right.
$$


Si nous négligeons les gradients verticaux des vitesses horizontales $u, v$, c'est-à-dire si nous considérons qu'il s'agit de courants de masses dans lesquels la vitesse horizontale ne dépend pas de la profondeur, et que les dénivellations $\zeta$ restent faibles, l'équation de continuité :

$$
\operatorname{div}(\vec{V}) \equiv k\left(a_{x}+v_{y}\right)+w_{z}=0
$$

s'écrira ici :

$$
u_{x}+v_{y}+(1 / h)\left[\lambda(\zeta / k)+h_{x} u+h_{y} v\right]=0
$$

ou :

$$
(h u)_{x}+(h v)_{y}+\lambda(\zeta / k)=0
$$

En éliminant $n$ et $v$ entre (1), (2), (3), nous obtenons l'équation déterminant $\zeta$, soit :

$\left.g h(\Delta \zeta-\Delta \varepsilon)+g\left[h_{x}-\gamma /(\varphi+\lambda) \cdot h_{y}\right]\left(\zeta_{x}-\varepsilon_{x}\right)+g[\gamma /(\varphi+\lambda)] . h_{x}+h_{y y}\right]\left(\zeta_{y}-\varepsilon_{i j}\right)=[\lambda /(\varphi+\lambda)]\left[(\varphi+\lambda)^{2}+\gamma^{2}\right]\left(\zeta / k^{2}\right)$

$\zeta$ étant détermince, $u$ et $v$ s'en déduisent par :

$$
\begin{aligned}
& {\left[(\rho+\lambda)^{2}+\gamma^{2}\right](u / k)=-g(\xi+\lambda)\left(\zeta_{x}-\varepsilon_{y}\right)-g_{\gamma}\left(\zeta_{y y}-\varepsilon_{y y}\right)} \\
& {\left[(\rho+\lambda)^{2}+\gamma^{2}\right](v / k)=+g \gamma\left(\zeta_{y}-\varepsilon_{x}\right)-g(\rho+\lambda)\left(\zeta_{y}-\varepsilon_{y y}\right)}
\end{aligned}
$$

\section{II. - SOLUTION DE DIVERS CAS PARTICULIERS}

Poincaré a mentionné comment il envisageait l'emploi de la méthode de Fredholm (par les équations intégrales) ou de celle de Ritz (par la réduction à un calcul des variations) pour la résolution d'une équation aux dérivées partielles du type (I) $\left(^{*}\right)$. Cependant, si l'on tient compte de la forme tourmentée du contour des océans et du relief de leurs fonds, l'application numérique de ces méthodes soulève encore de grosses difficultés, même avec les moyens puissants apportés par la technologie moderne des machines à calculer.

A défaut d'une solution générale, nous avons recherché pour certains cas particuliers soit un développement mathématique, soit un procédé arithmétique ou analogique fournissant une réponse aux problèmes rencontrćs. Nous indiquons ci-après quelques résultats de cette recherche.

Précisons d'abord que notre étude s'est limitée au cas des oscillations sinusoïdales. Désignant par a la pulsation, nous avons ainsi $\lambda=j \sigma$. En explicitant les solutions, nous négligeons systématiquement le facteur temporel $e^{j \sigma^{*}}$, qui sera supposé sous-entendu (**).

\section{1. - Zone géographique restreinte; profondeur uniforme; rotation uni-} forme; absence de frottement; absence de potentiel générateur. sent à :

Avec $k=1, h=$ constante, $y=$ constante, $\lambda=j 5 ; 0=0, \varepsilon=0$ les équations (I), (II), (III) se rédui-

$$
\begin{array}{rlrl}
g h \Delta \zeta+\left(\sigma^{2}-\gamma^{2}\right) \zeta & =0 \\
u=\frac{g}{\sigma^{2}-\gamma^{2}}\left(j \sigma \zeta_{x}+\gamma \zeta_{y}\right) ; & v & =\frac{g}{\sigma^{2}-\gamma^{2}}\left(-\gamma \zeta_{x}+j \sigma \zeta_{y}\right)
\end{array}
$$

La forme des équations amène à distinguer trois cas selon que $|\gamma / \sigma|$ est :

inférieur à 1: rotation lente ou hypo-critique,

égal à 1 : rotation critique,

supérieur à 1 : rotation rapide ou hyper-critique.

(*) Réf. «Leçons de Mécanique céleste » par Henri Poincaré, tome ITI, chap. X.

$\left({ }^{*}\right)$ Ainsi la solution $\zeta(x, y)$ représentera l'oscillation sinusoïdale, qu'on expliciterait complètement en ne retenant que la partie réelle du produit $\zeta(x, y) e^{j \sigma t}$. 
Sous la forme ci-dessus, le système d'équations perd d'ailleurs sa signification dans le cas critique, sur lequel nous reviendrons plus loin.

Pour $|\% / \sigma|<1$ (rotation lente), une solution élémentaire s'exprime par les relations $\left(^{\star}\right)$ :

$$
\left\{\begin{aligned}
\zeta & =A e^{j \alpha} \exp \left(\sqrt{\frac{\sigma^{2}-\gamma^{2}}{g h}} \cdot \operatorname{sh} \eta \cdot \mathrm{Y}\right) \cdot \exp \left(-j \cdot \sqrt{\frac{\sigma^{2}-\gamma^{2}}{g h}} \cdot \operatorname{ch} \eta \cdot \mathrm{X}\right) \\
\frac{\mathrm{U}}{\zeta} & =\sqrt{\frac{g}{h}} \cdot \frac{\sigma \operatorname{ch} \eta+\gamma \operatorname{sh} \eta}{\sqrt{\sigma^{2}-\gamma^{2}}} ; \quad \frac{\mathrm{Y}}{\zeta}=j \sqrt{\frac{g}{h}} \cdot \frac{\sigma \operatorname{sh} \eta+\gamma \operatorname{ch} \eta}{\sqrt{\sigma^{2}-\gamma^{2}}}
\end{aligned}\right.
$$

avec:

$$
\mathrm{X}=x \cdot \cos \theta+y \cdot \sin \theta \quad \mathrm{U}=u \cdot \cos \theta+v \cdot \sin \theta
$$

et :

$$
\mathrm{Y}=-x \cdot \sin \theta+y \cdot \cos \theta \quad \mathrm{V}=-u \cdot \sin \theta+v \cdot \cos \theta
$$

Pour $|\gamma / \sigma|>1$ (rotation rapide), une solution élémentaire s'exprime par les relations :

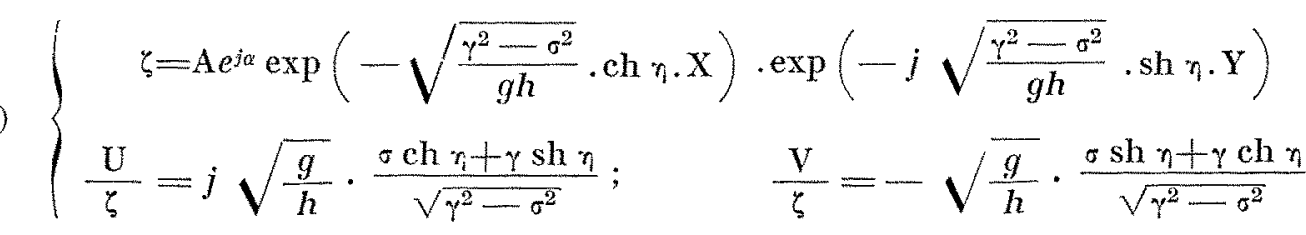

avec la même définition de $\mathrm{X}, \mathrm{Y}, \mathrm{U}, \mathrm{V}$.

Chacune de ces solutions élémentaires est définie par deux constantes complexes :

- le factear A $e^{j \alpha}$ qui en détermine l'amplitude (A) et la phase initiale ( $\alpha$ );

- le paramètre $\theta+j \eta$, qui, par sa partie réelle, $(\theta)$, détermine la direction et le sens de la propagation et, par le coefficient de $j,(\eta)$, détermine la forme de la crête, la célérité et la longueur de l'onde.

Quelques cas particuliers sont intéressants à signaler.

ONDE CYLINDRIQUE (ou « $\dot{a}$ crête horizontale 》) SANS ROTATION.

Pour $\gamma=0$ et $\eta=0$, les formules (L) deviennent :

$$
\left\{\begin{array}{l}
\zeta=A e^{j \sigma} \cdot \exp \left(-j \frac{\sigma}{\sqrt{g h}} \cdot \mathrm{X}\right) \\
\frac{\mathrm{U}}{\zeta}=\sqrt{\frac{g}{h}}, ; \mathrm{V} \equiv 0
\end{array}\right.
$$

ONDE A CRETE EXPONENTELLE SANS ROTATION.

L'introduction d'une rotation $\gamma \neq 0$ n'est nullement indispensable pour retrouver une onde $\dot{a}$ crête exponentielle: il suffit en effet, si $y=0$, de faire $\eta \neq 0$ dans les formules (L) pour trouver :

$$
\left\{\begin{array}{l}
\zeta=\mathrm{A} e^{j a} \cdot \exp \cdot\left(\frac{\sigma}{\sqrt{g h}} \cdot \operatorname{sh} \eta \cdot \mathrm{Y}\right) \exp \left(-j \frac{\sigma}{\sqrt{g h}} \operatorname{ch} \eta_{\cdot} \mathrm{X}\right) \\
\frac{\mathrm{U}}{\zeta}=\sqrt{\frac{g}{h}} \cdot \operatorname{ch} \eta ; \quad \frac{\mathrm{V}}{\zeta}=j \sqrt{\frac{g}{h}} \operatorname{sh} \eta \neq 0
\end{array}\right.
$$

nul.

Notons que, à la différence du cas précédent, cette onde comporte un courant traversier non

(*) Cette solution s'établit aisément en cherchant à déterminer $m$ et $n$ pour que $\zeta=\exp (m x+n y)$ vérifie l'équation en $\zeta$; on trouve la condition $m^{2}+n^{2}=(1 / g h)\left(\gamma^{2}-\sigma^{2}\right)$, à laquelle on satisfait en prenant $m= \pm \sqrt{(1 / g h)\left(\gamma^{2}-\sigma^{2}\right)} \cdot \cos (\theta+j \eta)$ et $n= \pm \sqrt{(1 / g h)\left(\gamma^{2}-\sigma^{2}\right)} \cdot \sin (\theta+j \eta)$. 
ONDE CYLINDRIQUE (ou « $\dot{a}$ crête horizontale 》) EN ROTATION LENTE.

Par contre [et cette solution a été signalée par Poincaré ( ${ }^{\star}$ ) et par Sverdrup (**)], dans le cas d'une rotation lente $(0 \neq|\gamma / \sigma|<1)$, on peut définir une onde $\dot{\alpha}$ crête horizontale, mais à courant transversal non nul, en faisant $\eta=0$ dans les formules (L) :

$$
\left\{\begin{array}{l}
\zeta=\mathrm{A} e^{j \alpha} \cdot \exp \left(-j \sqrt{\frac{\sigma^{2}-\gamma^{2}}{g h}} \cdot \mathrm{X}\right) \\
\frac{\mathrm{U}}{\zeta}=\sqrt{\frac{g}{h}} \cdot \frac{\sigma}{\sqrt{\sigma^{2}-\gamma^{2}}} ; \quad \frac{\mathrm{V}}{\zeta}=j \sqrt{\frac{g}{h}} \cdot \frac{\gamma}{\sqrt{\sigma^{2}-\gamma^{2}}} \neq 0
\end{array}\right.
$$

ONDE dE KELVin EN ROTATION LeNTE.

Pour annuler le courant traversier $V$ dans les relations (L), il faut prendre $\eta_{\text {tel }}$ que th $\eta=-(\gamma / \sigma)$; on obtient ainsi londe dite « de Kelvin »:

$$
\left\{\begin{array}{c}
\zeta=A e^{j a} \exp \left(-\frac{\gamma}{\sqrt{g h}} \mathrm{Y}\right) \exp \left(-\frac{j \sigma}{\sqrt{g h}} \mathrm{X}\right) \\
\frac{\mathrm{U}}{\zeta}:=\sqrt{\frac{g}{h}} ; \quad Y \equiv 0
\end{array}\right.
$$

ONDE A CRÊTE EXPONENTIELLE ET A PROTIL HORIZONTAL EN ROTATION RAPIDE.

En faisant $\eta=0$ dans (R), on trouve :

$$
\left\{\begin{array}{c}
\zeta=\mathrm{A} e^{j \alpha} \exp \left(-\sqrt{\frac{\gamma^{2}-\sigma^{2}}{g h}} \mathrm{X}\right) \\
\frac{\mathrm{U}}{\zeta}=j \cdot \sqrt{\frac{g}{h}} \cdot \frac{\sigma}{\sqrt{\gamma^{2}-\sigma^{2}}} ; \quad \frac{\mathrm{V}}{\zeta}=-\sqrt{\frac{g}{h}} \cdot \frac{\gamma}{\sqrt{\gamma^{2}-\sigma^{2}}}
\end{array}\right.
$$

ONDE DE KELVIN EN ROTATION RAPIDE.

Pour l'onde définie par les formules (R), la propagation s'effectue dans la direction OY. Pour annuler la composante $U$, qui est alors transversale, il faut prendre $\eta$ tel $q u e$ th $\eta=-(\sigma / \gamma)$; londe, que nous baptiserons encore « onde de Kelvin », correspond à la représentation :

$$
\left\{\begin{array}{l}
\zeta=A e^{j \mu} \cdot \exp \left(-\frac{\gamma}{\sqrt{g h}} \mathrm{X}\right) \cdot \exp \left(j \cdot \frac{\sigma}{\sqrt{g h}} \mathrm{Y}\right) \\
\mathrm{U}=0 ; \quad \frac{\mathrm{V}}{\zeta}=-\sqrt{\frac{g}{h}}
\end{array}\right.
$$

Certaines ondes élémentaires, ou certaines combinaisons simples de deux ou plusieurs de ces ondes, fournissent la solution de problèmes relatifs à l'oscillation de l'eau dans des domaines de forme géométrique élémentaire (canal de largeur constante, rectangle, etc.). Parmi les combinaisons linéaires de deux ondes élémentaires, Fichot a baptisé les ondes suivantes :

ONde DE POINCARÉ DE PREMIÈre ESPÈce (en rotation lente seulement) :

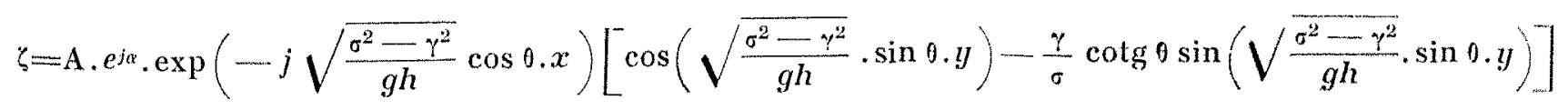

(*) « Théorie des Marées » (Gauthier-Villars, Paris, 1910), §66.

(*) « The Oceans » (N.Y. Prentice Hall, 1946), formules (XIV, 30) et (XIV, 31). 
ONDE DE POINGARÉ de DEUXIÈME ESPÈcE (en rotation lente) :

$$
\zeta=\mathrm{A} \cdot e^{j \alpha} \cdot \exp \left(-\sqrt{\frac{\sigma^{2}-\gamma^{2}}{g h}} \cdot \operatorname{sh} \eta \cdot x\right)\left[\sin \left(\sqrt{\frac{\sigma^{2}-\gamma^{2}}{g h}} \cdot \operatorname{ch} \eta y\right)+j \frac{\sigma}{\gamma} \operatorname{cth} \eta \cdot \cos \left(\sqrt{\frac{\sigma^{2}-\gamma^{2}}{g h}} \cdot \operatorname{ch} \eta \cdot y\right)\right]
$$

Onde de PoIncaré de deuxiÈme espèce (en rotafion rapide) :

$$
\zeta=\mathrm{A} \cdot e^{j \alpha} \cdot \exp \left(-\sqrt{\frac{\gamma^{2}-\sigma^{2}}{g h}} \cdot \operatorname{ch} \eta \cdot \mathrm{X}\right)\left[\sin \left(\sqrt{\frac{\gamma^{2}-\sigma^{2}}{g h}} \cdot \operatorname{sh} \eta \cdot \mathrm{Y}\right)+j \frac{\sigma}{\gamma} \operatorname{th} \eta \cdot \cos \left(\sqrt{\frac{{\gamma^{2}}^{2}-\sigma^{2}}{g h}} \cdot \operatorname{sh} \eta \cdot \mathrm{Y}\right)\right]
$$

D'une manière générale, la solution d'un problème comportant, sur une frontière plus ou moins compliquée, des conditions du type de Dirichlet, de Neumann, de Cauchy ou d'un type mixte s'établira en sommant un ensemble fini ou infini de solutions élémentaires.

\section{Rotation CRITIQUe.}

Revenons sur les cas particuliers oủ $\gamma= \pm \sigma$; pour fixer les idées, supposons $\gamma=+\sigma$. Dans ce cas, la méthode d'élimination de $u, v$, entre les équations (1), (2) et (3) tombe en défaut, car les équations (1) et (2) ne peuvent être résolues en $u$ et $v$ (le déterminant des coefficients de $u$ et de $v$ dans ces deux équations est nul); on peut montrer que, dans ce cas, l'ensemble des solutions est plus étendu que dans le cas général. La dénivellation $\zeta$ est assujettie - en plus des données aux limites - à la condition d'être une fonction analytique de la variable complexe $x$ - jy, soit $\left(^{*}\right)$ :

$$
\zeta=\mathrm{F}(x-j y)
$$

La définition des composantes $u$ et $v$ de la vitesse fait alors intervenir une autre fonction analytique, non plus de la variable $x-j y$, mais de la variable conjuguée $x+j y$, soit $\mathrm{G}(x+j y)$; on $\mathrm{a}\left({ }^{\star \star}\right)$ :

$$
\begin{aligned}
& u=\frac{\partial}{\partial x}\left(\frac{-g}{2 j \sigma} \mathrm{F}+\mathrm{G}\right)-\left(\frac{\partial}{\partial x}-j \frac{\partial}{\partial y}\right)\left(\frac{j \sigma}{2 \pi h} \iint \mathrm{F}^{\prime} \cdot \log r \cdot d x^{\prime} d y^{\prime}\right) \\
& v=\frac{\partial}{\partial y}\left(\frac{-g}{2 j \sigma} \mathrm{F}+\mathrm{G}\right)+\left(\frac{\partial}{\partial x}-j \frac{\partial}{\partial y}\right)\left(\frac{\sigma}{2 \pi h} \iint \mathrm{F}^{\prime} \cdot \log r \cdot d x^{\prime} d y^{\prime}\right)
\end{aligned}
$$

avec :

$$
\mathrm{F}^{\prime}=\mathrm{F}\left(\boldsymbol{x}^{\prime}-j y^{\prime}\right) \quad \text { et } \quad x=\sqrt{\left(x^{\prime}-x\right)^{2}+\left(y^{\prime}-y\right)^{2}}
$$

Marée statique de la troisiéme sorte.

Les formules ci-dessus montrent que avec $\zeta \equiv 0$ on peut avoir $\overrightarrow{u l, \vec{v}})=\overrightarrow{\mathrm{grad}}[\mathrm{G}(x+j !)] \neq 0$, la fonction $G$ ne dépendant que des données aux limites.

On obtient une marée statique au sens donné à ce mot par Poincaré, qui entendait par là que, dans une telle marée, la forme de la surface libre n'est pas affectée par le mouvement. Rappelons que Poincaré distinguait la " marée statique de la première sorte», dans laquelle il n'y a pas de courant, et la «marée statique de la deuxième sorte», dans laquelle les courants sont permanents. La définition ci-dessus se rapporte à une "troisième sorte 》de marée statique dans laquelle les courants sont alternatifs (de pulsation $\sigma \neq 0$ ).

(*) Telle est la condition de compatibilité des équations (1) et (2).

(* *) Les formules exprimant $u$ et $v$ a l'aide des fonctions $\mathrm{G}$ et $\mathrm{F}$ s'établissent à partir des relations $h\left(u_{x}+v_{y}\right)+j \sigma \zeta=0$ et $h\left(v_{a}-u_{y}\right)-\sigma \zeta=0$ qui définissent le rotationnel et la divergence du vecteur vitesse en fonction de la dénivellation. La fonction $\mathrm{F}(x-j y)$ est celle qui représente $\zeta$. La fonction $\mathrm{G}(x+j y)$ est introduite par l'intégration (cf. Lamb : « Hydrodynamics», \$ 154, et Villat : «Leçons sur la théorie des tourbillons », chap. II). 


\section{2. - Zone géographique restreinte; profondeur uniforme; rotation uni- forme; frottement fonction linéaire de la vitesse du courant; absence de potentiel générateur.}

Avec $k \equiv 1, h=$ constante, $\gamma=$ constante, $\lambda=j \sigma, p=$ constante, $\varepsilon=0$, l'équation (I) se déduit à :

$$
g h \Delta \zeta-\frac{j \sigma}{\rho+j \sigma}\left[(\rho+j \sigma)^{2}+\gamma^{2}\right] \zeta=0
$$

La solution élémentaire s'écrit :

$$
\zeta=\mathrm{A} e^{j \alpha} \cdot \exp (-r \mathrm{X} \operatorname{ch} \eta+s \mathrm{Y} \operatorname{sh} \eta) \cdot \exp [j(-s \mathrm{X} \operatorname{ch} \eta-r \mathrm{Y} \operatorname{sh} \eta)]
$$

avec :

$$
\begin{aligned}
& \mathrm{X}=x \cos \theta+y \sin \theta ; \quad \mathrm{Y}=-x \sin \theta+y \cos \theta \\
& \text { et } r^{2}=\frac{\sigma}{g h} \cdot \frac{\sqrt{\left(\sigma^{2}+\rho^{2}\right)\left[(\sigma+\gamma)^{2}+\rho^{2}\right]\left[(\sigma-\gamma)^{2}+\rho^{2}\right]}-\sigma\left(\sigma^{2}-\gamma^{2}+\rho^{2}\right)}{2\left(\sigma^{2}+\rho^{2}\right)} \\
& s^{2}=\frac{\sigma}{g h} \cdot \frac{\sqrt{\left(\sigma^{2}+p^{2}\right)\left[(\sigma+\gamma)^{2}+p^{2}\right]\left[(\sigma-\gamma)^{2}+p^{2}\right]}+\sigma\left(\sigma^{2}-\gamma^{2}+p^{2}\right)}{2\left(\sigma^{2}+q^{2}\right)} \\
& \text { Si } \gamma^{2}<\sigma^{2}+\rho^{2} \text { (rotation lente) } \\
& \text { on a } r^{2}<s^{2} \\
& \text { Si } \quad \gamma^{2 i}>\sigma^{2}+p^{2} \text { (rotation rapide) } \\
& \text { on a } r^{2}>s^{2}
\end{aligned}
$$

mais le cas intermédiaire $\left(\gamma^{2}=\sigma^{2}+p^{2}\right)$, n'est pas un cas critique, car on a alors :

$$
r^{2}=s^{2}=\frac{\sigma p}{g h} \neq 0
$$

La prise en compte dans les équations d'un frottement non nul ( $\neq 0)$ fait donc disparaitre toutes les singularités analytiques attachées au cas critique, tel que nous l'avons étudié dans l'hypothèse précédente $(\rho=0)$.

3. - (Cas schématique des marées littorales).

Zone géographique restreinte; profondeur non uniforme, mais pente du fond faible; rotation uniforme; frottement; absence de potentiel générateur.

Les hypothèses de ce schéma se traduisent par $k \equiv 1, h_{x} \# 0 \# h_{y}, \varepsilon=0$, ce qui conduit à l'équation aux dérivées partielles :

$$
g h \Delta \zeta-\frac{j \sigma}{\rho+j \sigma}\left[(\rho+j \sigma)^{2}+\gamma^{2}\right] \zeta=0
$$

à laquelle on peut, en vue des applications numériques, substitucr un système d'équations aux différences finies qui Iui soit pratiquement cquivalent et qui permette l'emploi d'un processus de calcul « arithmétique».

On peut encore rechercher une solution « analogique » des problèmes relatifs aux marces littorales par la mise en œuvre d'un modele réduit hydraulique. Mais, en l'absence d'une rotation d'entrainement imposée à la plate-forme qui le porte, le terme en $\gamma$ se trouve pratiquement éli- miné de l'équation qui détermine les dénivellations de la surface libre du modele, soit :

$$
g h^{\prime} \Delta \zeta-j \sigma\left(\sigma^{\prime}+j \sigma\right) \zeta=0
$$

II est cependant possible d'identifier (e) et $\left(e^{\prime}\right)$ en prenant :

$$
h^{\prime}=h \frac{1}{1-\left[\gamma^{2} /\left(\rho^{2}+\sigma^{2}\right) \mid\right.} \quad o^{\prime}=0 \frac{1+\left[\gamma^{2} /\left(\rho^{2}+\sigma^{2}\right)\right]}{1-\left[\gamma^{2} /\left(\rho^{2}+\sigma^{2}\right)\right]}
$$

c'est-à-dire en « distordant» convenablement la profondeur $h^{\prime}$ et la rugosité $\rho^{\prime}$ que l'on reproduira sur le modèle.

La représentation qui en résultera pour le champ des dénivellations $\zeta$ sera correcte si les conditions aux limiles le sont. Mais le champ des vitesses sera lui-même distordu, selon la corrélation :

$$
\left(\rho^{\prime}+j \sigma\right) \overrightarrow{V^{\prime}}=[(\rho+j \sigma)+\gamma \cdot \vec{v} \Lambda] \vec{V} \quad(*)
$$

$\left.{ }^{*}\right) \vec{v}$ désignant le vecteur unitaire porté par la verticale $M Z$, lopérateur géométrique $\vec{y} \Lambda$ est un verseur droit dans le plan horizontal. 
dont il faudra tenir compte dans les conditions aux limites, là où elles sont données en fonction des vitesses : ainsi une paroi imperméable dans la nature se trouvera représentée par une source de débit fluctuant.

\section{4. - (Cas schématique des marées océaniques). \\ Zone géographique étendue; profondeur non uniforme, mais pente du fond faible; rotation non uniforme; absence de frot- tement; action du potentiel générateur.}

Avec $h_{s} \# 0 \# h_{y}, \lambda=j \sigma$ et $\varphi=0$, l'équation (I) devient :

$$
g h(\Delta \zeta-\Delta \varepsilon)+\frac{\sigma^{2}-\gamma^{2}}{k^{2}} \zeta=0
$$

Comme dans le eas précédent, les problèmes relatifs aux marées océaniques peuvent être abordés soit, "arithmétiquement », en substituant à (E) un système aux différences finies, soit encore, " analogiquement », par l'emploi d'un modèle réduit dont les dénivellations de la surface libre seront déterminées par l'équation :

$$
g h^{\prime}\left(\Delta \zeta^{\prime}-\Delta s^{\prime}\right)+\sigma^{2} \zeta^{\prime}=0
$$

qu'il est possible d'identifier à (E) par un ensemble approprié de distorsions non uniformes des échelles de simililude.

Essentiellement, l'échelle des profondeurs sera:

$$
\frac{h^{\prime}}{h}=\frac{k^{2} \sigma^{\prime 2}}{\sigma^{2}-\gamma^{2}}=k^{2 \theta-2} \cdot \frac{1}{1-\left(\gamma^{2} / \sigma^{2}\right)}
$$

(avec $\sigma / \sigma^{\prime}=0=$ échelle des temps, uniforme).

Cette échelle des profondeurs $\left(h^{\prime} / h\right)$ pourra être toute différente de l'échelle des dénivellations $\left(\zeta^{\prime} / \zeta\right)=\left(\varepsilon^{\prime} / \varepsilon\right)$, qui sera choisie beaucoup plus grande (jusqu'à 1000 fois plus grande), pour obtenir, sur le modèle, un rapport $\left(\zeta^{\prime} / \varepsilon^{\prime}\right)$ mesurable, alors que $(\zeta / \varepsilon)$, qui est de l'ordre de $10^{-4}$, n'est pratiquement pas mesurable dans la nature. La puissance apportée par l'action du potentiel $g_{\varepsilon}$ sur la marée $\zeta$ dans une aire S étant proportionnelle à $g_{\varepsilon} . S \zeta$, on réalisera une échelle uniforme des échanges moyens d'énergie en adoptant pour l'échelle $\left(\varepsilon^{\prime} \zeta^{\prime} / \varepsilon_{\zeta}\right)$ une loi de répartition inverse de celle qui définit l'échelle des aires $\left(S^{\prime} / S\right)=k^{2}$, c'est-à-dire en adoptant :

$$
\left(\zeta^{\prime} / \zeta\right)=\left(\varepsilon^{\prime} / \varepsilon\right)=m_{0}\left(k / k_{0}\right)^{-1}
$$

$m_{0}$ et $k_{0}$ étant des constantes.

Dans ces conditions, les champs des vitesses modèle $\left(\vec{V}^{\prime}\right)$ et nature $(\vec{V})$ se correspondront selon la relation :

$$
\overrightarrow{V^{\prime}}=m_{0} k_{0} k^{-2}\left[1+\left(\gamma^{\prime} j \sigma\right) \vec{\vee} \Lambda\right] \overrightarrow{\mathrm{v}}
$$

Reste à préciser comment, sur le modèle, on peut représenter l'action du potentiel générateur qui figure dans les équations par les termes en $\varepsilon$. Sans nous étendre sur cette question, indiquons seulement que des fiuctuations du fond d'amplilude $\varepsilon$ donnent à l'eau la même impulsion que des fluctuations du niveau d'équilibre de même amplitude $\varepsilon$, la disposition relative de la surface libre actuelle et de sa position d'équilibre étant la même dans l'un et l'autre cas.

On peut, dans une premiere approximation, prendre pour $\varepsilon$ un harmonique donné du potentiel générateur luni-solaire (calculable à partir des éphémérides astronomiques); le terme correspondant du potentiel du bourrelet peut être calculé, en vue d'une seconde approximation, à partir des résultats d'une première mesure.

Notons toutefois que l'analogie proposée ne vaut que si les échelles auxquelles elle conduit sont acceptables. En particulier, on doit avoir $\left(h^{\prime} / h\right)>0$, e'est-à-dire $|\gamma / \sigma|<1$, ce qui limite la zone géographique représentée aux deux parallèles eritiques, de latitudes $L_{w}= \pm \operatorname{Are} \sin (\sigma / 2 \omega)$, soit $\mathrm{L}_{c}= \pm 75^{\circ}$ pour le cas de l'onde semi-diurne lunaire $\mathrm{M}_{2}$. Dans chacune des zones hyper-critiques polaires, de latitude $\mathrm{L}>\left|\mathrm{L}_{v}\right|$ on $\mathrm{L}<-\left|\mathrm{L}_{c}\right|$, l'analogie pourrait cependant être transposée sur un modèle animé d'une rotation plus rapide que l'image de la rotation $\omega$ à l'échelle des temps, 0 .

Nous ne nous étendrons pas sur ces extensions possibles. Nous noterons seulement que l'analogie sur un modèle fixe s'adapte parfaitement à l'étude de l'onde solaire semi-diurne $S_{2}$ pour laquelle les parallèles limites se réduisent aux deux pôles $\left(\mathrm{L}_{c} \# \pm 90^{\circ}\right)$ et très bien à l'étude de l'onde lunaire semi-diurne $\mathrm{M}_{2}\left(\mathrm{~L}_{c} \# \pm 75^{\circ}\right)$ qui est presque partout la composante prépondérante de la marée. Car, si ce n'est par l'ouverture (tronçonnée par l'Islande el les Feroë), entre Ecosse et Groënland, qui met en communication l'Atlantique-Nord avec l'Océan Arctique, la quasi-totalité des océans profonds, situés dans le zone hypo-critique $\left(|\mathrm{L}|<75^{\circ}\right)$, ne peurent échanger de débits importants avee les zones hypercritiques polaires, où l'action directe du potentiel générateur est d'ailleurs très minime.

Pour les ondes diurnes $\left(\mathrm{L}_{c} \# 30^{\circ}\right.$ ou $\left.29^{\circ}\right)$, l'emploi de modèles tournants figurant les zones polaires s'imposerait sans doute, ce qui soulèverait d'ailleurs le délicat problème du raccordement entre modèle fixe et modèle tournant sur le parallẻle critique, pour lequel l'emploi brutal des formules (qui négligent le frottement) conduit à une profondeur infiniment grande sur l'un et l'autre modèles. 

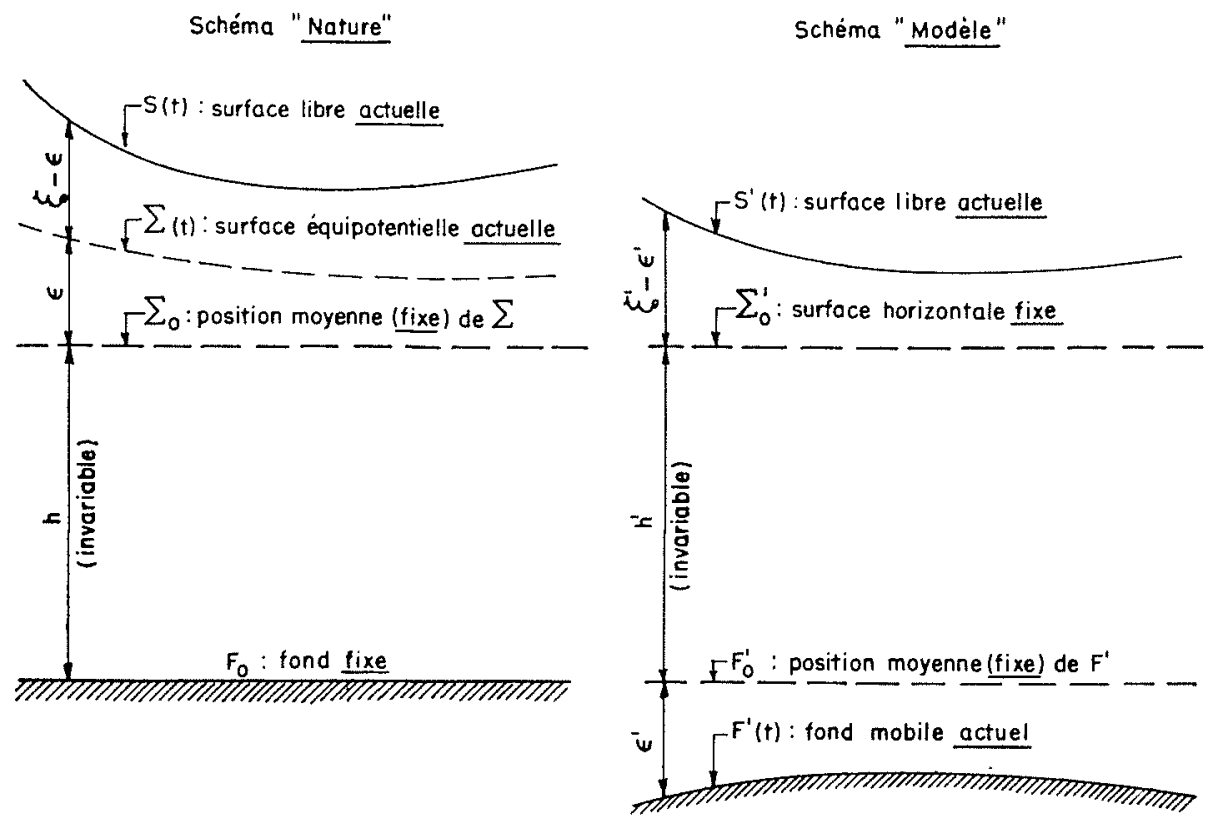

FIG. 2.

Quant aux ondes de plus longue période (semimensuelles, mensuelles, semi-annuelles, annuelles), il y a tout lieu de penser que les phénomènes d'inertie y peuvent être négligées et que, par suite, l'étude dynamique en serait vaine; la marée vraie à longue période est sans doute très proche de la marée statique $(\zeta \# \varepsilon)$.

\section{III. - CONCLUSION}

Les diverses méthodes, théoriques ou expérimentales, sommairement décrites ci-dessus, permettent - grâce aux techniques dont disposent les chercheurs modernes - d'approcher la solution des problèmes que pose le phénomène des marées.

Des considérations globales, d'ordre énergétique, donnent à penser que, grosso modo, les ondes océaniques sont stationnaires et que l'énergie apportée aux océans profonds par l'action du potentiel générateur (puissance moyenne de l'ordre du milliard de $\mathrm{kW}$ ) est presque intégralement transmise aux mers littorales, peu profondes, où les courants intenses des ondes dérivées, à caractère le plus souvent progressif, la dissipent par frottement, viscosité et turbulence. Mais cette interprétation est encore discutable.

On pourra sans doute, si cela s'avère utile, consacrer les moyens nécessaires à la réalisation des études dont nous avons exposé les principes, pour savoir dans quelle mesure les marées océaniques se rapprochent du schéma de Whewell avec le seul Océan Antarctique générateur d'ondes progressives dérivées vers l'Océan Indien, ie Pacifique et l'Atlantique, ou de celui de Harris avec sa partition, un tantinet arbitraire, du domaine océanique en bassins de résonance.

\section{DISCUSSION}

Président : M. Gibrat

M. le Président rappelle que M. Vantroys vient de présenter au Comité Technique, avec d'ailleurs, quelques indications supplémentaires, l'essentiel de la thèse de doctorat ès-sciences qu'il a soutenue très brillamment le 23 février dernier.

A propos d'une remarque de M. Vantroys, M. le Président indique que, si les Patagons pensaient que les marées venaient de la lune, c'est qu'ils croyaient être sur une mer littorale, et non océanique, puisque l'appli- cation des équations de $M$. Vantroys relatives aux mers littorales donne une prépondérance à l'effet Iunaire.

M. Vantroys précise que la Patagonie est, en fail, très près des fonds océaniques (Baie de San-José).

M. Hupner se demande si le géogmaphe qui avait des idées un peu bizarres sur les marées ne serait pas Paganel, embarqué, d'après Jules Verne, avec les enlants du Capitaine Grant. 
M. Gotgenhem indique que c'est M. Marcel BoLl, qui dans un de ses ouvrages, attribue à Sarcey la phrase: «Les paysans bretons (il s'agissait des Bretons et non des Patagons) sont si arriérés qu'ils attribuent ì la lune une influence sur les marées $\gg$. M. Boll et son collaborateur n'ont pu lui donner la référence exacte; il ne l'a pas retrouvée non plus dans la collection des feuillets de Sarcey publiés dans le Temps.

M. Govgenhein félicite M. Vantroys de l'impulsion nouvelle qu'il a donnée aux recherches sur les marées. L'enseignement de Poincaré avait suscité quelques travaux (thèses de Jaeger, de Blondel, de Bertrand) qui découlaient directement de ses idées, mais d'une manière peut-être trop stricte. M. Vantroys, sous la nécessité des besoins pratiques, a prolonge les travaux de Poincares dans une vole encore peu explorée. Ses recherches sur l'application des modèles réduits aux marées sont on grande partie nonvelles et en tout cas beaucoup plus poussées que tout ce qui avait été fait antẻrieurement. Leur réalisation est éminemment souhaitable.

N. Kravtchenko souligne l'intérêt de l'exposé de M. Vantroys et se propose do préciser les différences entre les cas hypo-critique, critique et hyper-critique, signalés par M. VANTnoxs et qui correspondent à trois régimes physiques distincts. Il souhaite que l'étude analytique des solutions du type non elliptique soit poursuivie.

M. VANTroYs ajoute que la distorsion des profondeurs, sur la carte de Mercator, qu'il a représentée, est relative au cas hypo-critique, mais que la zone hyper-critique peut être représentée sur un modèle tournant, en introduisant un coefficient $\gamma^{\prime}$ non nul dans l'équation du modèle, de manière à identifier les équations « modèle » et « nature » sans être conduit à attribuer des valeurs négatives au rapport $h^{\prime} / h$.

D'autre part, en réponse à deux questions de M. HupNER, M. Vantroys rappelle le sens de ses notations et précise la notion de «latitude critique».

$\gamma$ est le double du produit de la vitesse angulaire de la terre par le sinus de la latitude $(=2 \omega \sin L)$

$\sigma$ est la pulsation $[=(2 \pi / \mathrm{T})]$, $\mathrm{T}$ étant la période $(12 \mathrm{~h}$. 25 pour l'onde semi-diurne lunaire, $12 \mathrm{~h}$. pour l'onde semi-diume solaire; ce serait de 6 secondes à 12 secondes pour la houle).

Quand on décrit une oscillation sinusoïdale, on peut poser :

$$
x=a \cos (\sigma t-\varphi)
$$

$x$ étant la hauteur variable et a l'amplitude de l'oscillation. D'autre part, la latitude critique est celle, définia par son sinus, pour laquelle on a $\gamma=\sigma$
Cette latitude critique varie donc d'une onde composante à une autre. Une première étude descriptive des marées peut se limiter à l'onde semi-diurne lanaire $M$. c'est à cette onde que se rapporte l'exemple graphique projeté, on complète ensuite, si nécessaire, par l'étude des autres composantes.

M. Grbert demande à M. VaNthoys s'il a eu commais. sance des travaux de la commission chargée aux PaysBas, sous la direction du Professeur Lorentz, d'étudier la modification de la marée littorale produite par la fermeture du Zuydersee. M. Gıbert pense qu'il s'est agi surtout d'un problème de propagation de la marée dans un système complexe de chenaux et il ne croit pas qu'il ait été tenu compte de l'influence de la force de Coriolis; par ailleurs, le terme de frottement avait été pris proportionnel à la vitesse.

II. VANTroys répond qu'il n'a eu que quelques echos des travaux du Professeur Lorentz; et que d'autre part, Poincaré, sans s'étendre sur le cas du frottement, a représenté dans une partie de son exposé le frottement par un terme proportionnel à la vitesse.

En réponse à la question posée par M. Giberr, M. Krnavtchenko eite un rapport inédit, en hollandais, do J.M. Burgers sur les marées de la mer du Nord et les travaux de Van Dantzig, d'Amsterdam et de ses élèves Hveltkamp et Laurier. Ces travaux ont fait l'objet d'uns communication (á paraitre) aux Actes du Congrès de Mécanique de Bruxelles, en 1956.

M. Vasmors ajoute que les applications numériques les plus poussées sont, à sa connaissance, celles de l'océanographe allemand Walter Hansen : il a calculé la marée à l'intérieur de la mer du Nord à partir de données aux limites.

M. Chapouther remarque que la prise en compte des frottements supprime le passage par la solution critique. Cette circonstance analytique traduit sans doute une différence physique. Quelle est sa signification? Comment, dans le cas de rugosité, passe-twon d'un régime à l'autre? (1)

M. Kravtchenro indique qu'au point de vue mathématique, la prise en compte du frottement revient à introduile dans l'équation différentielle du second ordre à coefficient constant, qui est l'image la plus simple du phénomène, un terme proportionnel à la dérivíe première.

(1) N. Vantroys a fait au cours de la discussion de la communication de M. BonNefille une réponse $\dot{a}$ cette question, basée sur les résultats obtenus par ce dernies.

\section{E R R A T U M}

\section{N*A 1957.}

Page 298, $1^{\text {re }}$ colonne, ligne 6 en remontant :

au lieu de $g \mathrm{~S} / a=\alpha$, lire $: a / g \mathrm{~S}=\alpha$.

$2^{\circ}$ colonne, figure 4 , au point $b .5$ :

au lieu de $-48,50$, lire : $-48,05$.

Page 299, $1^{\text {re }}$ colonne, ligne 7 :

an lieu de «l'extrémité $C$ », lire: « de l'extrémité A». 